\title{
Right Suprarenal Vein
}

National Cancer Institute

\section{Source}

National Cancer Institute. Right Suprarenal Vein. NCI Thesaurus. Code C53131.

A vein that drains blood from the right adrenal gland into the inferior vena cava. 